\title{
Largest non-unique subgraphs
}

The reduction number $r(G)$ of a graph $G$ is the maximum integer $m \leq|E(G)|$ such that the graphs $G-E, E \subseteq E(G),|E| \leq m$, are mutually non-isomorphic, i.e., each graph is unique as a subgraph of $G$. We prove that $r(G) \leq \frac{|V(G)|}{2}+1$ and show by probabilistic methods that $r(G)$ can come close to this bound for large orders. By direct construction, we exhibit graphs with large reduction number, although somewhat smaller than the upper bound. We also discuss similarities to a parameter introduced by Erdős and Rényi capturing the degree of asymmetry of a graph, and we consider graphs with few circuits in some detail. 